\title{
Laparoscopic Inguinal Hernia Repair by Transabdominal Preperitoneal Mesh Hernioplasty (TAPP), A Prospective Study
}

\author{
Pankaj Saxena ${ }^{1}$, Kalpeshkumar Patel $^{2}$ \\ ${ }^{1}$ Professor \& Head, ${ }^{2} 3 r d$ year PG Resident, Department of General Surgery, Geetanjali Medical College and \\ Hospital, Udaipur, Rajasthan.
}

\begin{abstract}
Background: Hernia is not a disease of modern society; its occurrence was found during early 16th century and it was recognized as a surgical disease by Praxagoras of Kos because of its demand for some sort of life-saving treatment. In general, hernia is described as a protrusion of contents of abdominal cavity through a weakness or opening in the abdominal muscle wall. Laparoscopic hernia repair has emerged as an effective alternative method for treating inguinal hernias. It has several significant advantages over the tension-free open repair now in use. In this report we summarize our laparoscopic hernia repair results and Recommendations.
\end{abstract}

Method: The study was conducted in the Department of General Surgery, Geetanjali Medical College, Udaipur, from November 2017 to November 2019. It was a prospective, observational study on the patients of uncomplicated inguinal hernias who underwent an elective laparoscopic inguinal hernia repair. The following data was collected prospectively: age, sex, duration of surgery, intra-operative complications, postoperative pain, postoperative complications, hospital stay and recurrence.

Results: A total 30 patients were operated during study period and total number of hernia repair were 36 , with their ages renged from 22 to 80 year with mean age of 52.6year. All patents were male.The average duration of operation was $55.33 \pm 17.02$ minutes. The average length of hospital stay was 3.06day. The main complication in our study were postoperative seroma formation. One patient required conversion to open with zero recurrence till date.

Conclusion: Laparoscopic TAPP hernia repair has proven to be an efficient method for the treatment of groin hernias at our institution.

Keywords: Inguinal hernia, Laparoscopic Transabdominal preperitoneal approach.

\section{Introduction}

Hernia is not a disease of modern society; its occurrence was found during early 16th century and it was recognized as a surgical disease by Praxagoras of Kos because of its demand for some sort of lifesaving treatment. ${ }^{(1)}$ In general, hernia is described as a

\section{Corresponding author:}

\section{Dr. Kalpeshkumar Patel}

3rd year PG resident, Department of General Surgery, Geetanjali Medical College and Hospital, Udaipur, Rajasthan. Mobile - 7300344656

Email - patel.kkp.kalpesh@gmail.com protrusion of contents of abdominal cavity through a weakness or opening in the abdominal muscle wall. The mechanism behind such opening is still under debate in the course of anatomical defect or connective tissue disorder.

Based on the location on abdominal wall, hernias have been classified into various types which include inguinal, umbilical, and femoral canal regions. The incidence of inguinal hernias $(75 \%)$ is more compared to umbilical (9.5\%), incisional (6.2\%), femoral $(2.7 \%)$ and other types including spigelian, hiatal, or epigastric $(8.6 \%)$. $^{(2)}$ There are two types of inguinal hernia, direct and indirect, which are defined by their relationship to the inferior epigastric vessels. ${ }^{(3)}$ 
Recently laparoscopic hernia repairs become more popular. The basic anatomical principles of laparoscopic herniorrhaphy were first described by Spaw in 1991 based on human cadaveric dissections. ${ }^{(4)} \mathrm{He}$ coined the term "triangle of doom" delineating the region between the vas deferens and the spermatic vessels. In Transabdominal Preperitoneal Repair after entering the peritoneal cavity by laparoscopy the preperitoneal space is entered, sac dissected and reduced. Mesh is used to cover the defect by anchoring it to the Cooper's ligament and conjoint tendon. This is the most commonly performed laparoscopic repair.

\section{Materials and Method}

The study was conducted in the Department of General Surgery, Geetanjali Medical College, Udaipur, from November 2017 to November 2019. It was a prospective, observational study on the patients of uncomplicated inguinal hernias who underwent an elective laparoscopic inguinal hernia repair. The approval from the ethics committee of geetanjali medical college and a signed informed consent were obtained from the patients. All patients, above 18 years of age with uncomplicated inguinal hernia were included in the study. Complicated hernias, patients unfit for general anaesthesia were excluded. Operations were undertaken by a single surgeon of our team and all patients were followed up for a period of one year post operatively. The following data was collected prospectively: age, sex, duration of surgery, intra-operative complications, postoperative pain, postoperative complications, hospital stay and recurrence.

Post-operatively, by evening round after assessment patients were allowed oral intake of fluid of all kind, until then they were given supportive maintenance intravenous fluids. Patients were advised and encouraged to ambulate and start their daily persuits as early as possible. Patients were observed and documented for post operative complication such as hematoma, seroma, wound sepsis during their stay in the hospital. Evaluation of the post operative pain was done using visual analogue scale (VAS)(1-10) and carefully documentation of the use of analgesics (oral/injectable), (NSAID/opioids) were done from immediate to second post operative day.

Patients were discharged on second post operative day, once free of complains and complications. At discharge, they were advised to come on $7^{\text {th }}$ day $\left(1^{\text {st }}\right.$ follow-up), and then after 15 day ( $2^{\text {th }}$ follow-up), and then after 21 day of surgery, ( $3^{\text {rd }}$ follow-up). Later on after 1 month of surgery ( $4^{\text {th }}$ follow-up) and after 3 months after surgery ( $5^{\text {th }}$ follow-up) and at 6 month ( $6^{\text {th }}$ follow-up) and after 1 year ( $7^{\text {th }}$ follow-up).

\section{Inclusion Criteria}

1. Patients above 18 years of age.

2. Unilateral/Bilateral/Direct/Indirect/Noncomplicated/Completely reducible/Incomplete inguinal hernia (proven by clinical examination and abdominal ultrasound).

3. Patients of ASA grade 1 and 2.

4. Written informed consent from patient.

\section{Exclusion Criteria}

Patients with the following conditions are excluded from this study: Hernia with complications (irreducible hernia, partially reducible and strangulated hernia).

\section{Results}

A total of 30 patients were operated under general anaesthesia during the study period and the total number of hernias repaired were $36 ; 6$ patients had bilateral hernia repair. Patients included in the study were adults and their ages ranged from 22 to 80 years with a mean age of 52.6years with SD of 15.39 .

There were no female patients in our study. Out of 30 patients, 6 had bilateral hernias. Proportion of direct and indirect hernia is almost equal. The average duration of operation was $55.33 \pm 17.02$ minutes. It was observed that with experience, the learning curve for the laparoscopic inguinal hernia repair shortened that is from the first case which took 80 minutes the duration decreased to 30 minutes in a few later cases of laparoscopic repair (Table 1).

Table 1. Average duration of surgery.

\begin{tabular}{|l|l|l|l|l|l|}
\hline \multicolumn{7}{|l|}{ Average duration of surgery } \\
\hline & \multicolumn{3}{l|}{ Number of case } & $\begin{array}{l}\text { Total } \\
\text { cases }\end{array}$ \\
\hline & $\begin{array}{l}30- \\
39 \mathrm{~min}\end{array}$ & $\begin{array}{l}40- \\
49 \mathrm{~min}\end{array}$ & $\begin{array}{l}50- \\
60 \mathrm{~min}\end{array}$ & $>60 \mathrm{~min}$ & \\
\hline $\begin{array}{l}\text { Unilateral } \\
\text { cases }\end{array}$ & 4 & 8 & 6 & 6 & 24 \\
\hline $\begin{array}{l}\text { Bilateral } \\
\text { cases }\end{array}$ & 0 & 0 & 3 & 3 & 6 \\
\hline
\end{tabular}


The main complications in our study were post-operative seroma formation. Two(6.66\%) patients came with complain of swelling in inguinal region, Cough impulse over swelling was absent, ultra-sonography implied cord seroma which was ultra-sonography guided aspirated. One(3.33\%) patient presented with abdominal distention on first post operative day which was managed conservatively. There were no patients recorded with port site infection, funiculitis and scrotal hematoma. No recurrence recorded till date.(Table 2)

One (3.33\%) intra operative complication seen was inferior epigastric vessel injury which was managed by harmonic scalpel. Post operatively ecchymosis was seen on patient's lower abdominal wall and inguinal region which subsided spontaneously on $4^{\text {th }}$ follow up (on 1month) (Table 3).

Table 2.Post-operative complications $(\mathrm{n}=30)$.

\begin{tabular}{|l|l|l|l|}
\hline S.No. & Postoperative complication & No. of patients & Percentage \\
\hline 1 & Chronic pain & 1 & $3.33 \%$ \\
\hline 2 & Port site infection & Zero & $0.0 \%$ \\
\hline 3 & Funiculitis & Zero & $0.0 \%$ \\
\hline 4 & Urinary retention & 1 & $3.33 \%$ \\
\hline 5 & Scrotal hematoma & Zero & $0.0 \%$ \\
\hline 6 & Seroma & 2 & $6.66 \%$ \\
\hline 7 & Recurrence & Zero & $0.0 \%$ \\
\hline 8 & Abdominal distension & 1 & $3.33 \%$ \\
\hline 9 & Nerve injury & Zero & $0.00 \%$ \\
\hline 10 & Ecchymosis & 2 & $6.66 \%$ \\
\hline
\end{tabular}

Table 3.Intra-operative complications $(n=30)$.

\begin{tabular}{|l|l|l|l|}
\hline S.No. & Intraoperative complication & No. of patients & Percentage \\
\hline 1 & $\begin{array}{l}\text { Inferior epigastric vessels } \\
\text { injury }\end{array}$ & 1 & $3.33 \%$ \\
\hline
\end{tabular}

Table 4. Dose of analgesia required postoperatively $(n=30)$.

\begin{tabular}{|l|l|l|}
\hline No of doses & No of patients & Percentage \\
\hline First dose & 30 & $100 \%$ \\
\hline 2 dose & 10 & $33.33 \%$ \\
\hline$>2$ doses & 4 & $13.33 \%$ \\
\hline Total patients & 30 & $100 \%$ \\
\hline
\end{tabular}


Table 5. Duration of hospital stay $(n=30)$.

\begin{tabular}{|l|l|l|}
\hline Hospital stays (days) & No of patient & $\%$ \\
\hline 2 & 5 & $16.67 \%$ \\
\hline 3 & 20 & $66.67 \%$ \\
\hline 4 & 3 & $10 \%$ \\
\hline 5 & 2 & $6.67 \%$ \\
\hline Total & 30 & $100.00 \%$ \\
\hline Mean & 3.06 Days & \\
\hline SD & 0.73 & \\
\hline
\end{tabular}

The period of average stay is 72 hours which when split in "Time line" comes out as 24hours preoperative and 48hours postoperative.

\section{Discussion}

As a beginner in laparoscopic hernia surgeries following the common trend of going in first time, we also opted for an easier, simpler, neo orientation of surgical landmarks by adopting "TAPP" as our pioneering endeavour.

It is essential to mention that there were 6 patient operated for bilateral hernia, out of them two (2) patients were not aware about their contralateral hernia and even ultrasound examination reported normal for that contralateral side, yet during surgery by direct view, a small asymptomatic contralateral hernia was detected which was operated simultaneously as patient had given informed consent, which was asked routinely before TAPP in all patients. However, laparoscopic hernia surgery offers the possibility to repair both sides without additional trauma to the abdominal wall.

In this study, overall average duration to perform laparoscopic TAPP was $55.33 \pm 17.02$ minutes which included both unilateral and bilateral cases.(Table 1) This compared well with a study conducted by Chalkoo M.et $\mathbf{a l}^{(5)}$, in 2016 in which the overall mean operative duration in laparoscopic TAPP repair was 48.5 minutes. One more study conducted by Shamir O. Cawich. et al ${ }^{(6)}$ in 2013 showed that the average duration for unilateral repair was $68.5 \pm 10.4$ minutes and for bilateral repair $89 \pm 7.61$ minutes. In our study mean age of patients undergoing TAPP was $52.6 \pm 15$.4years. This is comparable with a large study conducted by Kapiris S.A. et $\mathbf{a l}^{(7)}$ in 2001 were the average age of patients undergoing TAPP hernia repair was 57years.

Most common types of hernia seen in our study were indirect inguinal hernia which comprised of around $52.77 \%$ (19/36), and the remaining $47.22 \%$ (17/36) were direct hernia. Our results are comparable to study conducted by Ayman et al. ${ }^{(8)}$ who found $52.8 \%$ of patients having unilateral indirect hernia. Another study conducted by Chalkoo M. et $\mathbf{a l}^{(5)}$ in 2016 and found $58.57 \%$ of patients having indirect hernia.

Injury to the inferior epigastric vessels are more common in TAPP while opening and closing of the peritoneum. There is one study conducted by Muschalla F. et $\mathbf{a l}^{(9)}$ in 2016 . They observed one case $(0.1 \%)$ of epigastric vessel injury and one case of testicular vessel injury. In our study, in 1 patient out of 36 hernias repair, there was injury to inferior epigastric vessels during creation of peritoneal flap. Intraoperatively bleeding was managed by harmonic scalpel (Table 3 ). Post operatively same patient presented with ecchymosis over right lower abdomen and inguinal region, which later resolved over the period of 1 month.

According to literatures, bowel lesions are described between $0 \%{ }^{(10)}$ to $4 \% .^{(11)}$ Injury to bowel is mostly during insertion of veress needle or by the electrocautery during adhesiolysis. To prevent that maximum care should be taken when gaining first access to the abdominal cavity and adhesions to the groin and hernial sac must not be taken down; in such cases an incision of peritoneum should be carried out slightly above the adhesion. In our 
study we did not record any case of bowel injury $0.0 \%$ (0 patient).

Out of total $6(20 \%)$ patients operated for bilateral inguinal hernia. In one (3.33\%) patient of bilateral inguinal hernia, intraoperatively we found on left side there is sliding inguinal hernia with herniation of sigmoid loop for that reason laparoscopic hernia was converted to open repair for that particular side to prevent bowel injury (Figure 1).

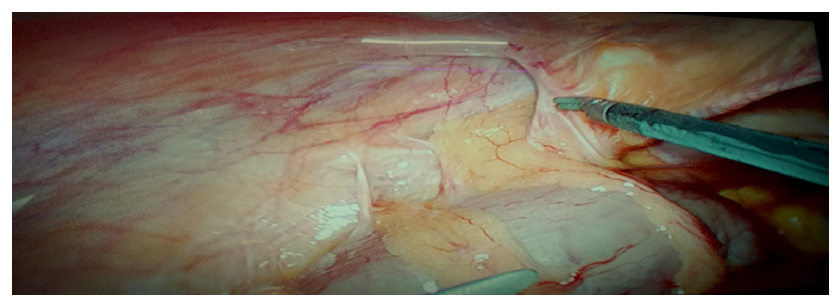

Figure 1: Showing left side sliding indirect inguinal hernia with herniation of sigmoid loop

While we are discussing postoperative complications According to the literatures, after laparoscopic hernia repair, haematomas are observed in $4.4 \%^{\left({ }^{(10)}\right.}$ and $13.1 \%^{(\mathbf{1 2})}$ of cases and seromas are observed in between $4.4 \%{ }^{(10)}$ and $12.2 \%^{(12)}$ of the cases. In our study we observed $2(6.66 \%)$ cases of seromas 1 in $7^{\text {th }}$ post operative day and other one in $14^{\text {th }}$ post operative day and they were managed by ultrasonography guided aspiration. We did not observe any case $(0.0 \%)$ of haematoma (Table 2$)$.

We saw urinary retention in $3.33 \%$ of our patient which is comparable to study literature. They reported about $3.5 \%^{(12)}$ and $3.8 \%{ }^{(10)}$ cases of urinary retention after laparoscopic hernia repair. According to literature review, occurrence of wound infection is reported between $0.5 \%{ }^{(10)}$ and $1.54 \%{ }^{(5)}$ of the cases. In our study no $(0.0 \%)$ case of wound infection is recorded. We recorded one case $(3.33 \%)$ of paralytic ileus in post operative period which was managed conservatively and recovery of gut activity happened in a 24 hours (Table 2).

Numerous randomized controlled trials have demonstrated that the recurrence rates are statistically comparable after open and laparoscopic repairs. ${ }^{(13-16)}$ There is one study conducted by Shamir O. et al (6) in 2013 were they recorded $(0.97 \%)$ recurrence. We had a zero recurrence recorded in the one year of our experience (Table 2). Majority patients were discharged after 48hours of operation, with average duration of hospital stay of 3 days which include preoperative period also. Median duration required for all patients to resume their normal work is 15 days. There is one study conducted by Chalkoo M. et $\mathbf{a l}^{(5)}$ in 2016, with majority of patients discharge within 24hours of surgery with median duration to return their normal activity was 16.1 days which is comparable.

Overall our initial experience with TAPP hernia repair was curiously interesting and encouraging as during this course we become familiar and later on confident in getting acquainted with new surgical anatomy and with proper choice of cases were able to achieve reasonably good results.

Our follow up may look a bit shorter as one year is all that we did, however we may expand the same in times to come.

\section{Conclusion}

With the experience of 30 laparoscopic hernia repair, we conclude that laparoscopic hernia repair may be a safe and effective alternative procedure to open inguinal hernia repair with faster recovery and earlier return to routine daily activity. It has fewer complications and recurrence rate.

Ethical Clearance: Taken from Human Research Ethical Committee

\section{Source of Funding: Self \\ Conflict of Interest: Nil}

\section{Bibliography}

1. Papavramidou NS, Christopoulou-Aletras H. Treatment of "hernia" in the writings of Celsus (First Century AD). World J Surg. 2005; 29: 1343-7.

2. Weber A, Garteiz D, Valencia S. Epidemiology of inguinal hernia: a useful aid for adequate surgical decisions. In: Bendavid R, Abrahamson J, Arregui M, Flament J, Phillips E, editors. Abdominal Wall Hernias: Principles and Management. New York, 2001.

3. Direct Inguinal Hernia. University of Connecticut. Retrieved May 6, 2012.

4. Spaw AT, Ennis BW, Spaw LP. Laparoscopic hernia repair: the anatomical basis. J Laparoendosc Surg. 1991;1(5):269-77.

5. Chalkoo, M., Mir, M.A. and Makhdoomi, H.(2016) Laparoscopic Transabdominal Preperitoneal Mesh Hernioplasty: A Medical College Experience. Surgical Science, 7, 107-113. 
6. Cawich S O, Mohanty S K, Bonadie K O, Simpson LK, Johnson PB, Shah S, et al. Laparoscopic Inguinal Hernia Repair in a Developing Nation: Short-term Outcomes in 103 Consecutive Procedures. J Surg Tech Case Report 2013;5:13-7.

7. Kapiris S.A, Brough W.A, et al. Laparoscopic transabdominalpreperitoneal (TAPP) hernia repair. A 7-year two-center experience in 3017patients. Surg Endosc (2001) 15:972-975.

8. Elwan, A.M., Abomera, M.A., Abo Al Makarem, M.A., and Abd Alhamed, H. (2013) Mohammedain: Laparoscopic Transabdominal Preperitoneal Repair versus Open Preperitoneal Mesh Repair for Inguinal Hernia. Journal of the Arab Society for Medical Research, 8, 38-42.

9. Muschalla F, Schwarz J, Bittner R. Effectivity of laparoscopic inguinal hernia repair (TAPP) in daily clinical practice: early and long-term result. R. SurgEndosc 2016; 30: 4985-4994.

10. Bittner R, Sauerland S, Schmedt CG. Comparison of endoscopic techniques vs. Shouldice and other open nonmesh techniques for inguinal hernia repair: a meta-analysis of randomized controlled trials. Surg Endosc. 2005;19:605-15.

11. Dedemadi G, Sgourakis G, Karaliotas C, Christofides T, Kouraklis G, Karaliotas C. Comparison of laparoscopic and open tension-free repair of recurrent inguinal hernias: a prospective randomized study. Surg Endosc. 2006;20:1099-104.

12. Schmedt CG, Sauerland S, Bittner R. Comparison of endoscopic procedures vs. Lichtenstein and other open mesh techniques for inguinal hernia repair. A meta-analysis of randomized controlled trials. Surg Endosc. 2005;19:188-99.

13. Dallas KB, Erovlich D, Choi JJ, Rosa JH, Lo C, Colon MJ, et al. Laparoscopic versus open inguinal hernia repair in octogenarians: A follow-up study. Geriatr Gerontol Int 2013;13:329-33.

14. Brooks DC. A prospective comparison of laparoscopic and tension-free open herniorraphy. Arch Surg 1994;129:361-6.

15. Anadol AZ, Ersoy E, Taneri F, Tekin E. Outcome and cost comparison of laparoscopic transabdominal preperitoneal hernia repair versus open Lichtenstein technique. J Laparoendosc Adv Surg Tech A 2004;14:159-63.

16. Tzovaras G, Symeonidis D, Koukoulis G, Baloviannis I, Georgopoulou S, Pratsas C, et al. Long-term results after laparoscopic transabdominal preperitoneal (TAPP) inguinal hernia repair under spinal anesthesia. Hernia 2012;16:973-9. 